\title{
Reconstruction of Intrahepatic Bile Ducts in Congenital Biliary Atresia
}

\author{
Tsuneo Chiba, Morio Kasai and Nobuaki Sasano* \\ The Second Department of Surgery and The Second Department \\ of Pathology*, Tohoku University School of Medicine, Sendai
}

Chiba, T., Kasai, M. and Sasano, N. Reconstruction of Intrahepatic Bile Ducts in Congenital Biliary Atresia. Tohoku J. exp. Med., 1975, 115 (2), 99-110 - Macroserial reconstruction of the main intralobular bile ducts was made in 7 cases of biliary atresia; 2 cases of type I, 1 case of type II and 4 cases of type III according to Kasai's classification. From the results of these reconstruction studies, it was confirmed that the main interlobular bile ducts are usually patent through the liver regardless of the type of atresia of the extrahepatic bile ducts. A microserial reconstruction of bile ducts and ductules of a small portal tract performed in one case disclosed that a number of ductules make a network around the main duct and have some communications with the main duct. These results were compatible with the fact that active excretion of bile was obtained in many postoperative patients with biliary atresia. As observed in one case of the present series, postoperative complication of severe ascending cholangitis seemed to be an important cause of destruction or disappearance of intrahepatic bile ducts, which has also seen in older infants with this disease without complication of cholangitis. In view of these facts the operation of an early stage of life is recommended in biliary atresia.___ congenital biliary atresia; reconstruction of intrahepatic bile ducts; ascending cholangitis

The pathogenesis of biliary atresia is not known. Although many kinds of study have been performed up to the present, the true cause of the disease is not clear. Recently, it has been reported by several authors that this disease may result from some inflammatory process of the liver and biliary tract during intrauterine life. Knowledges about the pattern of the bile ducts seem to be a prerequiste for studying the cause of this disease. The form of the intrahepatic biliary tree can be demonstrated by $\mathrm{x}$-ray films with contrast medium, a cast using resin, and histological reconstruction. The normal structure of intrahepatic biliary tree was previously described by Healey (1953). In the liver of biliary atresia, however, visualization of the bile ducts with contrast medium or resincast is practically impossible. Therefore, the technique of histological reconstruction is required for demonstration of bile ducts in the liver. There have been some reports on this subject, but most of them are concerned with the structure of bile ducts and ductules in the peripheral liver specimen. The present report describes the study of intrahepatic main bile ducts extending from the porta hepatis to the periphery of the liver in biliary atresia. 


\section{Materials and Methods}

Autopsy was performed in 7 cases of biliary atresia within 3 postmortal hours during the past four years at Tohoku University Hospital. The materials of the present study were obtained from these 7 cases. After the liver was removed from the abdomen, a specimen was excised en bloc from the porta hepatis to the periphery of the liver perpendicularly. The specimen was fixed in $10 \%$ neutral formalin. After fixation, serial cutting was made with an electric slicer horizontally to the porta hepatis in 6 cases and perpendicularly to the porta hepatis in 1 case. The thickness of each tissue block was about 2000 to $2500 \mu \mathrm{m}$. The number of the tissue block was variable, depending on the thickness of the liver (Fig. 1). Marks and numbers were written on the surface of each tissue block for confirmation of the site and direction. Following dehydration with alcohol, these blocks were transferred to the paraffin solvent which is miscible with the dehydrating agent. In 6 cases, xylene was used for paraffin solvent (paraffin imbedding). In the remaining case (Case 2), chloroform was used for paraffin solvent after the infiltration with ether and celloidin (celloidin-paraffin imbedding). After the process of paraffin imbedding these tissue blocks were attached to wooden blocks without distortion of the direction.

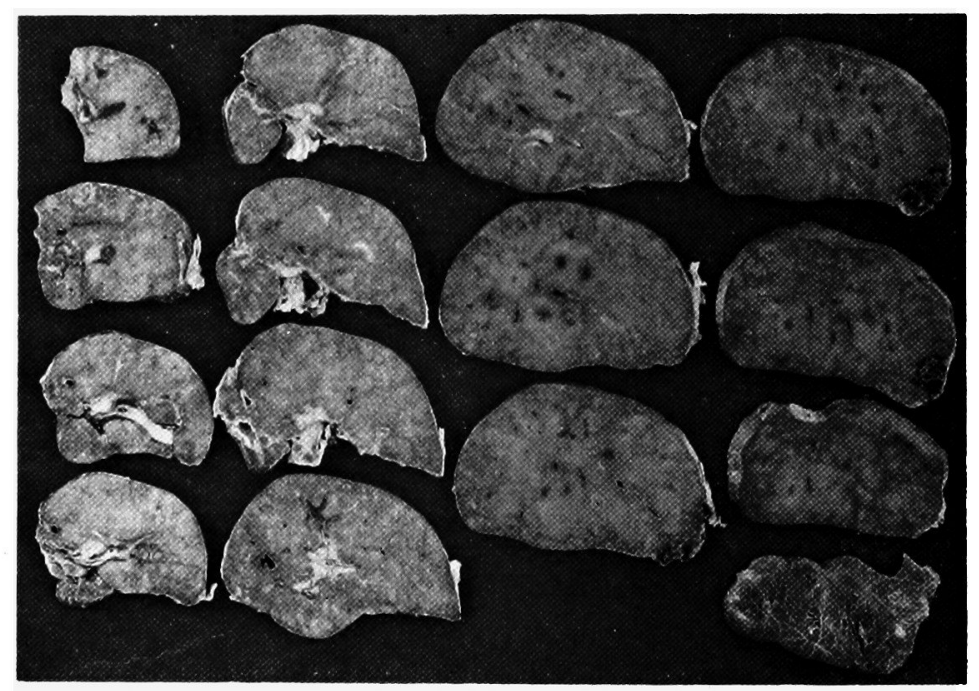

Fig. 1. A part of serial tissue blocks of the liver (Case 7).

Serial sections of $6 \mu \mathrm{m}$ in thickness were prepared from every celloidin-paraffin blocks of Case 2. In other cases, about 10 sections were obtained from every paraffin block. Each section was also $6 \mu \mathrm{m}$ in thickness. A large majority of the sections were stained with elastica-Masson stain for good discrimination of the cholangioles from other tissues such as vessels and the like. A small number of the sections were stained with hematoxylineosin. After staining, all sections were projected on tracing paper under high magnification. All the ducts and ductules within the portal tract demonstrated were traced. Thus the macroserial graphic reconstruction of large bile ducts and ductules in a small portal tract was made in addition to macroserial reconstruction of large bile ducts in Case 2 with a similar technique. 


\section{Results and Discussion}

According to Kasai's classification (Kasai 1966), gross findings of the extrahepatic bile duct were classified as type $I_{a}$ in Cases 1 and 4, as type $I_{b}$ in Case 2, as type $\mathrm{III}_{\mathrm{a}}$ in Case 6 and as type III $_{\mathrm{b}}$ in Cases 3,5 and 7 . Case 6 was male and the other cases were female. Three cases representing various types and two interesting cases are to be described in the following:

Case 1. Y.M., female. Her mother suffered from toxemia of pregnancy in the late pregnancy, but the delivery was normal. Body weight at birth was $2,850 \mathrm{~g}$. Jaundice had been continuously manifested after neonatal jaundice. She was admitted to the Second Department of Surgery, Tohoku University Hospital on January 18, 1971. The stool showed negative Schmidt's test for bilirubin and the total serum bilirubin was $14.6 \mathrm{mg} / 100 \mathrm{ml}$. The operation was performed on the 228 th day of life. The common bile duct was dilated and no communication with the duodenum was disclosed by intraoperative cholangiography. The type of the obstruction was Kasai's $I_{a}$. An external fistula with common bile duct was only made because of the poor general condition of the patient. The postoperative course was not good and serum bilirubin did not decrease. She died 18 days after operation. Autopsy revealed that the distal portion of the common bile duct was completely obliterated. The liver weighed $400 \mathrm{~g}$, which was firm in consistency. Histologically (Fig. 2), marked periportal fibrosis and moderate ductular proliferation in the periportal zone were seen. Hepatic cells were degenerated and many bile plugs were found in ductules in portal tracts and in bile canaliculi in lobules. On the cut surface adjacent to the porta hepatis, a comparatively large bile duct was found. The reconstruction of intrahepatic bile ducts (Fig. 4) demonstrated that the main interlobular bile duct was open along its

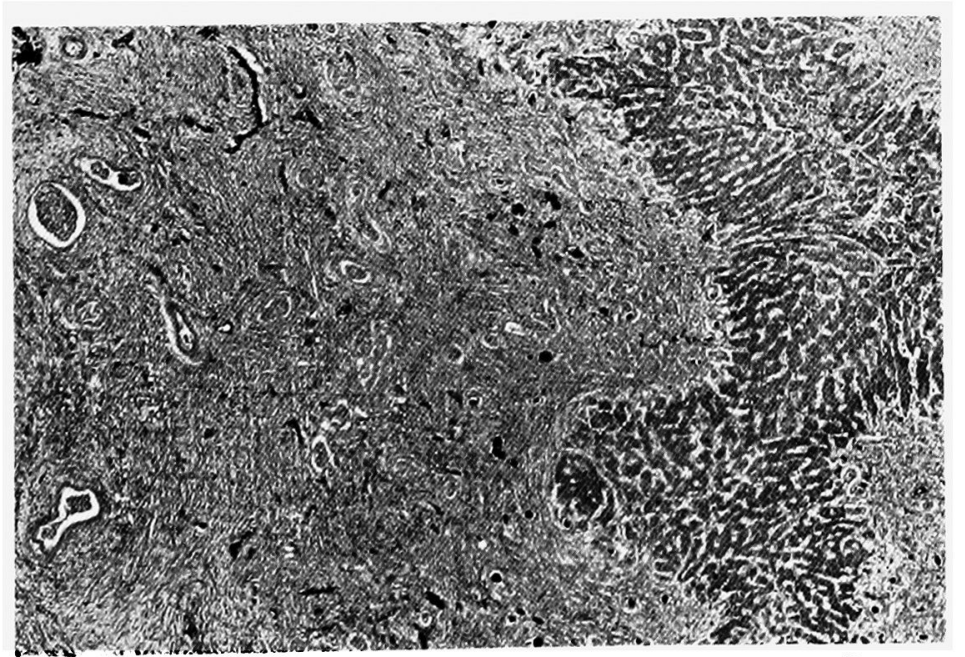

Fig. 2. Photomicrograph of the peripheral liver specimen in Case 1. 


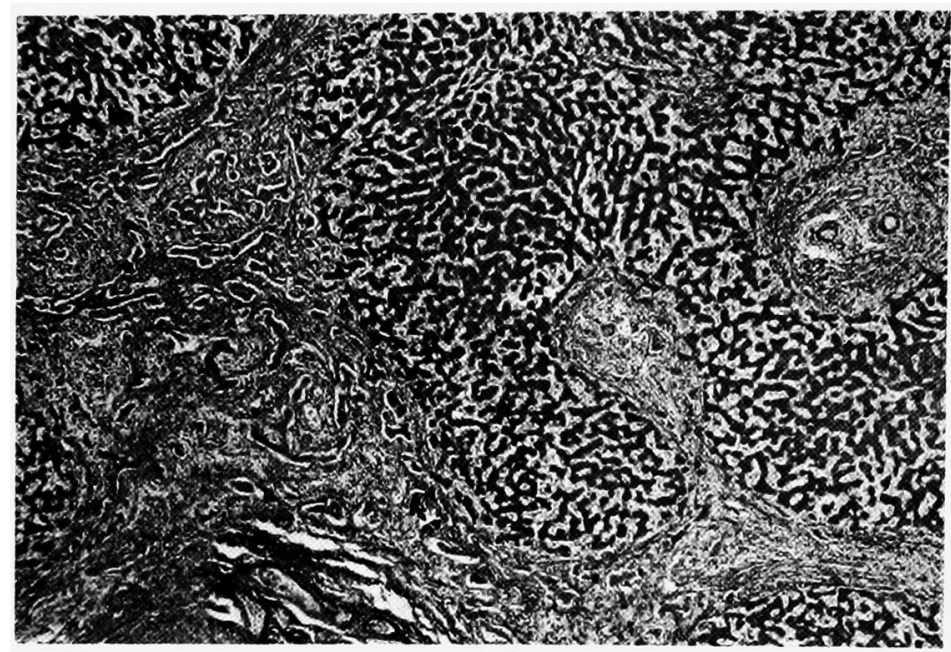

Fig. 3. Photomicrograph of the liver in Case 2.

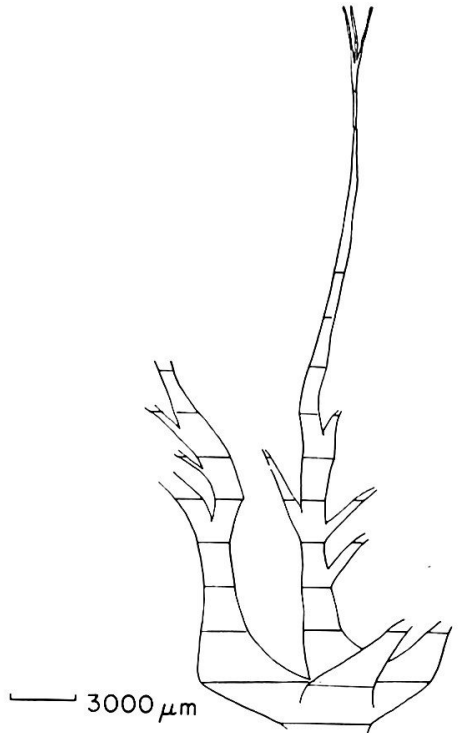

Fig. 4. A graphic reconstruction of some branches of intrahepatic bile duct in Case 1.

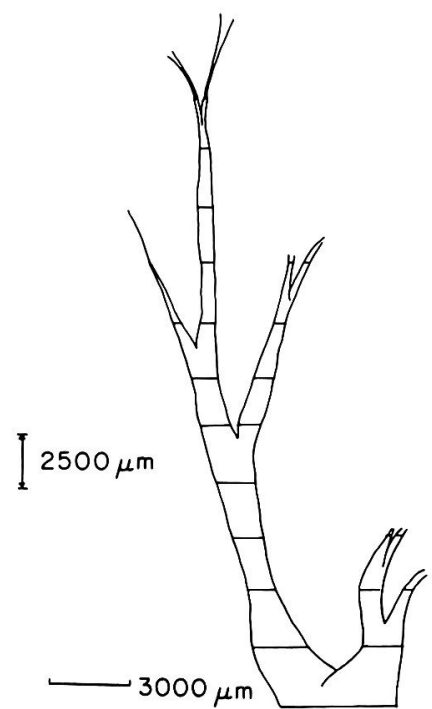

Fig. 5. A graphic reconstruction of some branches of intrahepatic bile duct in Case 2.

whole length through the liver. The reconstructed bile duct seemed to be the medial superior duct of the left lobe. The reconstructive findings of a bile duct in Case 4 which died 6 months of life were similar to those in Case 1.

Case 2. M.J., female. Her mother was in good health during the pregnancy. Body weight at birth was $3,000 \mathrm{~g}$. Persistent jaundice appeared after the neonatal jaundice once disappeared. She was admitted to our clinic on January 11, 1971. 


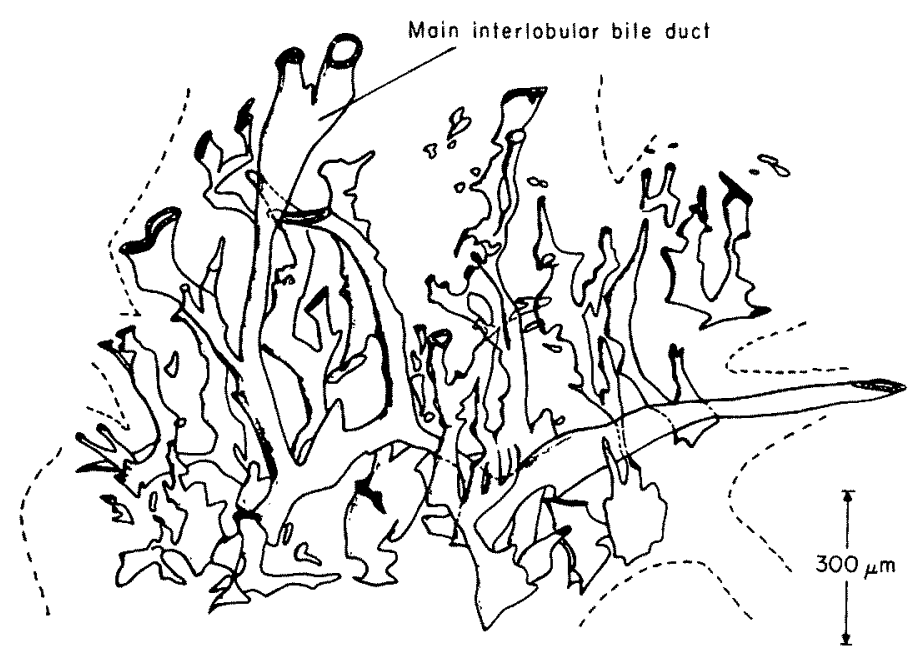

Fig. 6. A graphic reconstruction of the portal tract in Case 2.

Total serum bilirubin was $14.6 \mathrm{mg} / 100 \mathrm{ml}$. The operation was performed on the 89th day of life. Although the common bile duct and cystic duct were atretic, the common hepatic duct and both hepatic radicles were dilated and the contrast medium could be easily injected into the intrahepatic bile duct (Kasai's type $\mathrm{II}_{\mathrm{b}}$ ). Hepatic porto-jejunostomy with external fistula was performed (Sawaguchi et al. 1968). After the operation, bile excreted through this external fistula and jaundice diminished. However, she died of hematemesis and subsequent pulmonary edema on the 38th postoperative day. Autopsy revealed that hemorrhagic pneumonia and edema of both lungs and swelling of both kidneys existed. The liver weighed $350 \mathrm{~g}$, which was relatively firm. Histological study of the liver disclosed marked irregular periportal fibrosis (Fig. 3). Ductular proliferation was noticed in the periportal zones. Fig. 6 shows the microscopic reconstruction of bile duct and ductules in one block. This finding is consistent with that previously described by $\mathrm{Oh}-\mathrm{i}$ et al. (1969). The main draining duct was preserved and a number of ductules surrounded the main duct, occasionally connecting with it. Fig. 5 shows macroserial reconstruction of intrahepatic bile ducts of the same liver. The bile ducts were open along their whole length to the periphery of the liver.

Case 3. K.K., female. Her mother suffered from toxemia of pregnancy. Body weight at birth was $2,700 \mathrm{~g}$. Neonatal jaundice did not disappear. She was admitted to the Second Department of Surgery, Tohoku University Hospital on July 19, 1971. The total serum bilirubin was $10 \mathrm{mg} / 100 \mathrm{ml}$. The operation performed on 88th day of life revealed a total obliteration of extrahepatic bile ducts. The gallbladder was atrophic with a small lumen. The right hepatic duct, common hepatic duct and cystic duct were fibrous, while the left hepatic duct was not seen and classified as Kasai's III $_{\mathrm{b}}$. Fig. 7 shows a histological slide of macroserial 


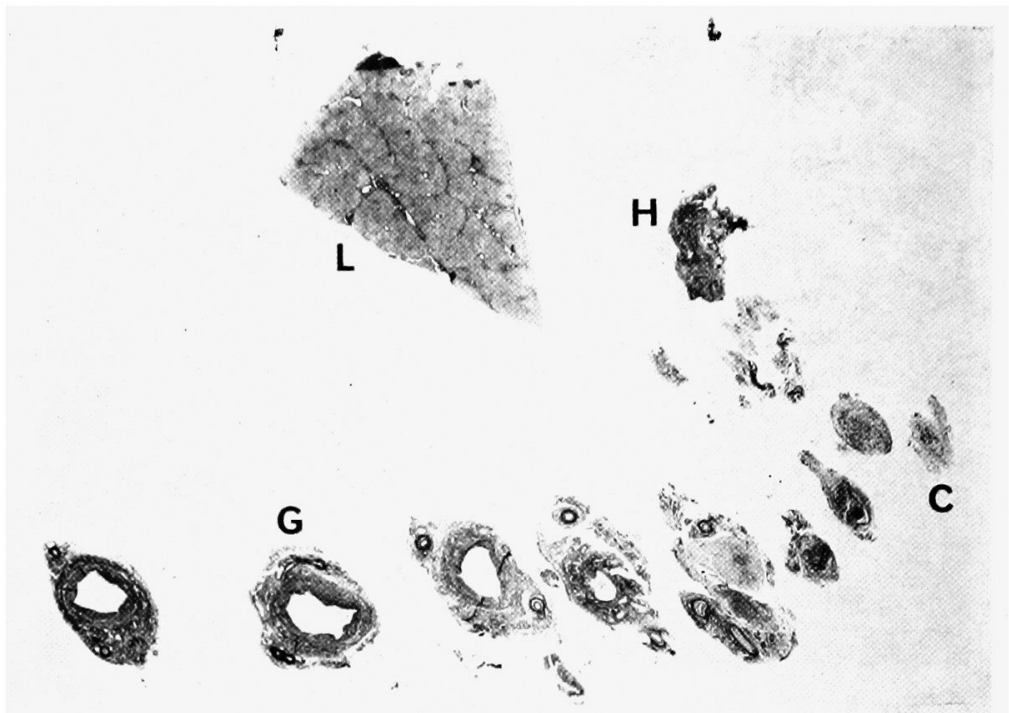

Fig. 7. Macroserial sections of the extrahepatic bile ducts in Case 3. C, common bile duct; G, gallbladder; $H$, hepatic duct (right); L, liver specimen.

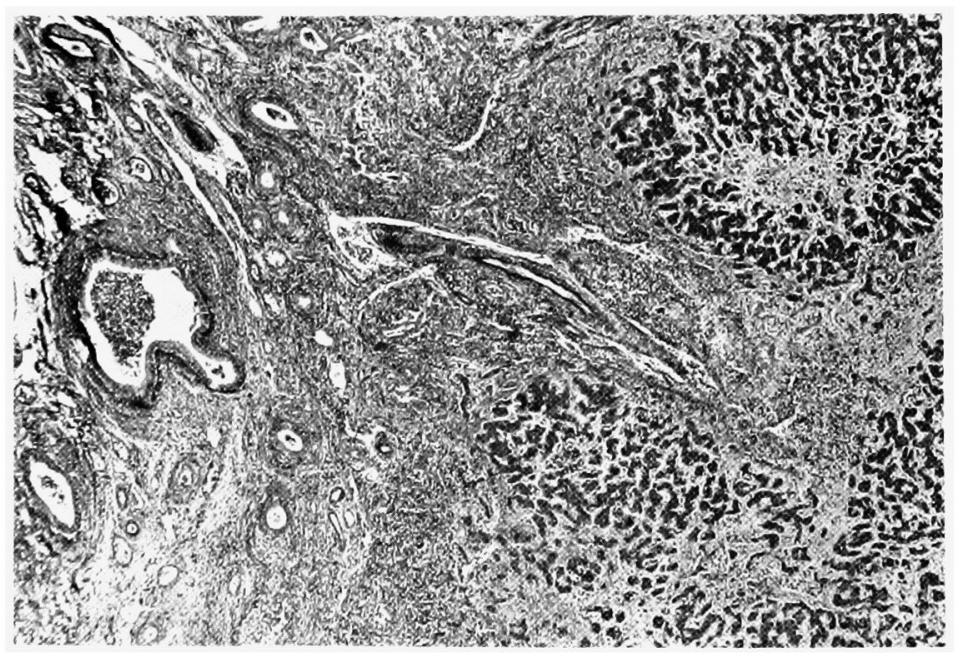

Fig. 8. Photomicrograph of the liver in Case 3.

sections of the extrahepatic bile duct removed from this patient. Hepatic portojejunostomy with Witzel-type fistula was performed. The tube jejunostomy was excised and double-barreled jejunostomy was made 10 days after the first operation because intestinal decompression through the tube was inadequate (Suruga 1970). After the second operation the general condition of the patient became worse and died of the anastomotic leakage 67 days after the first operation. The liver weighed $500 \mathrm{~g}$, which was firm in consistency at autopsy. As shown in Fig. 8, the liver histologically showed moderate ductular proliferation. An 


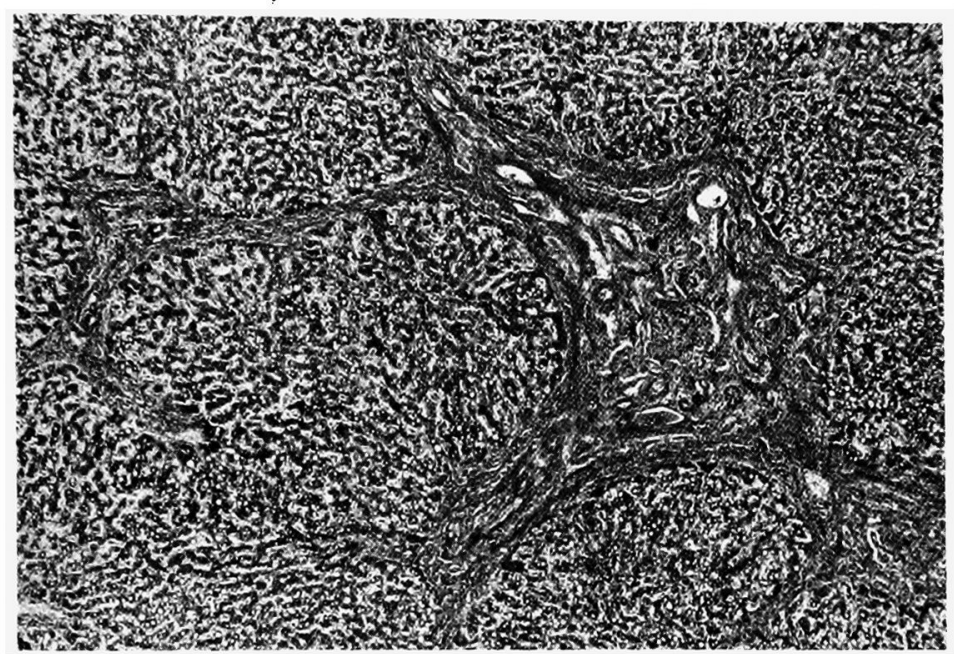

Fig. 9. Photomicrograph of the liver in Case 7.

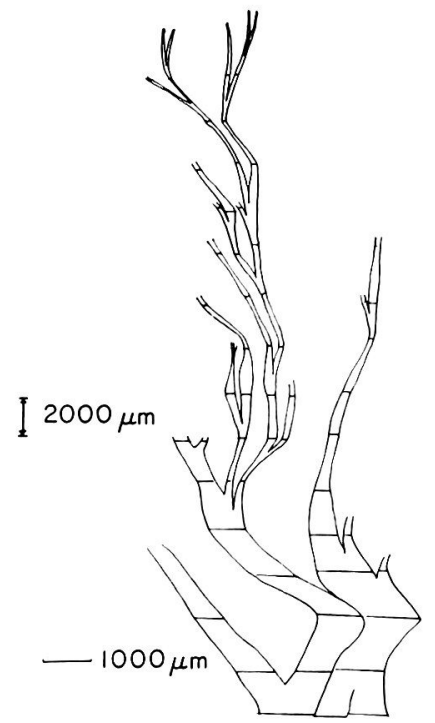

Fig. 10

Fig. 10. A graphic reconstruction of some branches of intrahepatic bile duct in Case 3.

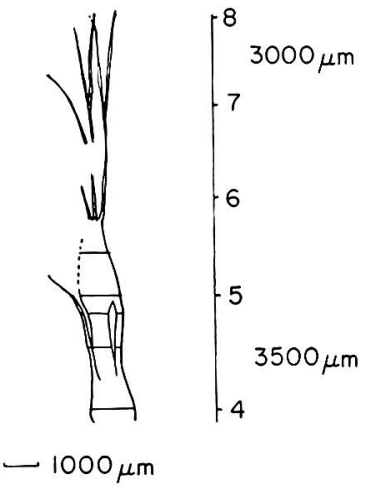

Fig. 11

Fig. 11. A graphic reconstruction of an intrahepatic bile duct in Case 5 .

interlobular bile duct was clearly recognized in a portal tract. Hepatic cells were moderately degenerated. A macroserial reconstruction of intrahepatic bile ducts indicated that the main bile ducts were patent along the whole length examined except at the porta heaptis (Fig. 10). Similar findings were observed in Case 6. 
Case 5. T.H.Y., female. The mother of this case suffered from common cold at 5 months of pregnancy, and exanthema at 6 months of pregnancy. She took some drugs during both periods of sickness. Body weight at birth was $3,600 \mathrm{~g}$. Jaundice had continued since her birth when she was admitted to Tohoku University Hospital on August 14, 1972. The total serum bilirubin was $10.3 \mathrm{mg} /$ $100 \mathrm{ml}$. The operation was performed on the 68th day of life. The type of the biliary atresia was Kasai's III $_{\mathrm{b}}$. Double-Y hepatic jejunostomy with an external fistula (Kasai et al. 1972) was carried out. Fig. 12 shows a histological slide of macroserial sections of the extrahepatic bile duct removed from this patient. The postoperative course was not good. Intestinal obstruction was complicated 23 days after the first operation and re-operation was necessary. However, the ascending cholangitis frequently occurred after the operation and no bile excretion was observed after the second operation. She died 5 months later because of ascending cholangitis. At autopsy, the liver was very firm, suggesting the biliary liver cirrhosis. Histological examination of the liver showed marked portal fibrosis linking with portal tracts each other (Fig. 13). Regenerative lobules with irregular arrangment of liver cells were seen (Fig. 13-A). B of Fig. 13 shows an intrahepatic bile duct at about $9 \mathrm{~mm}$ from porta hepatis which was considerably dilated and involved in acute inflammation. This bile duct was followed toward the periphery and found to decrease in size as shown C,D,E and F. In $\mathrm{E}$, the epithelial linking is completely destroyed and no lumen can be seen in

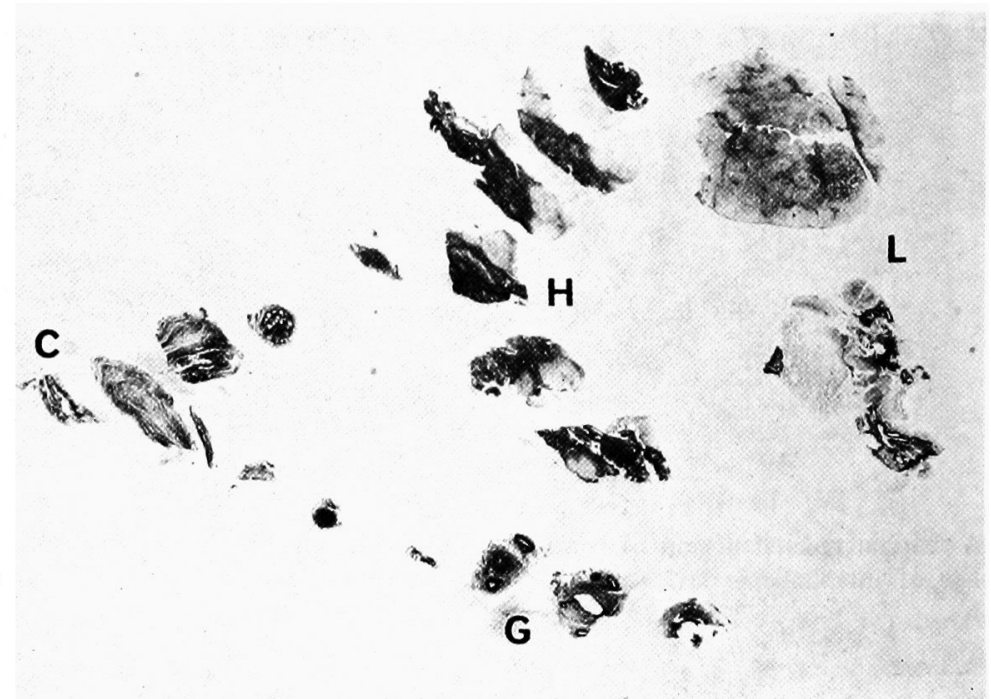

Fig. 12. Macroserial sections of the extrahepatic bile ducts in Case 5 .

$\mathrm{C}$, common bile duct; $\mathrm{G}$, gallbaldder; H, heaptic duct; L, liver specimen.

Fig. 13. Photomicrographs of the liver in Case 5 .

These pictures show the changing aspects of a main bile duct. The distance of each picture is about $2,500 \mu \mathrm{m}$. 


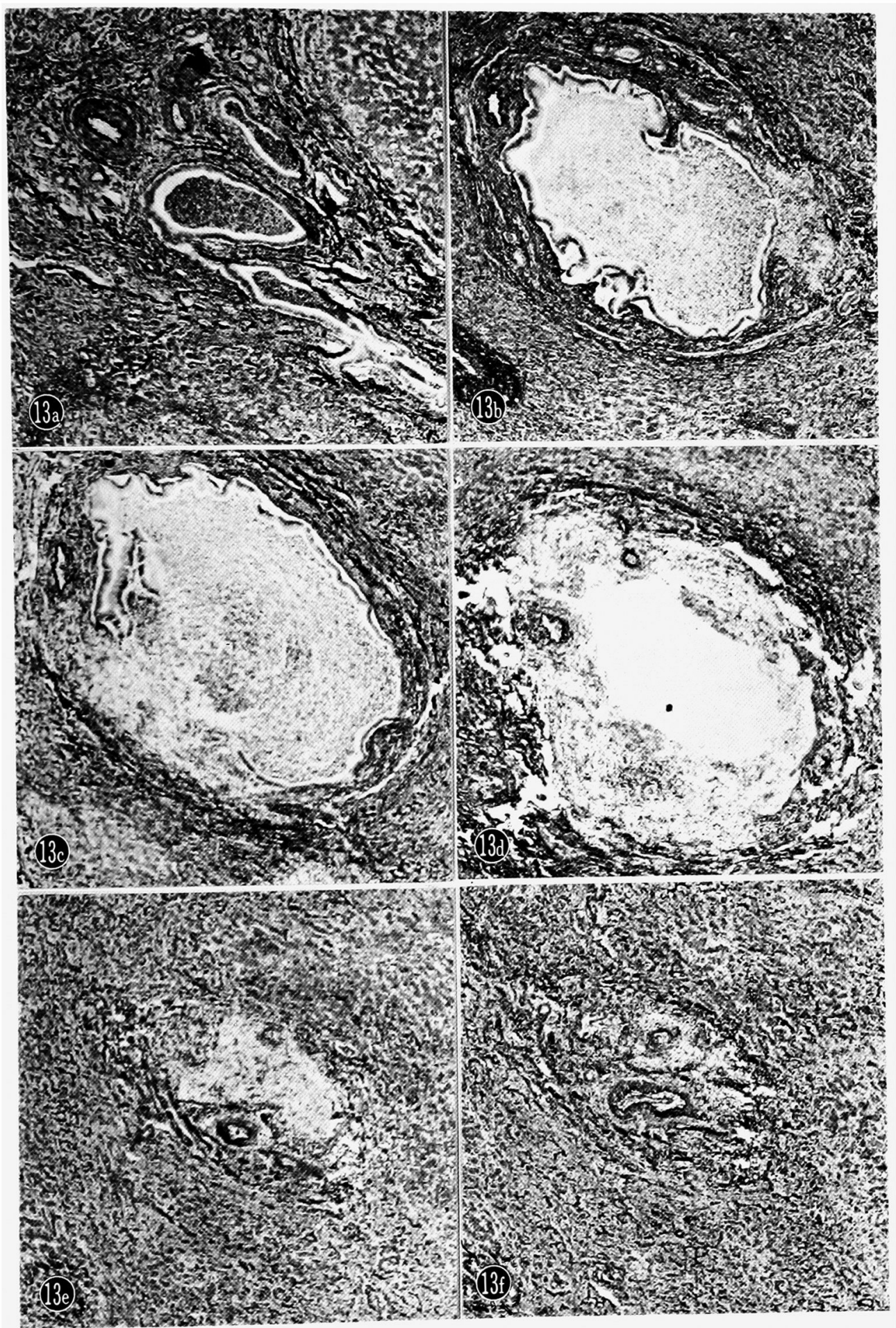


F any more. The macroserial sections of this portion of bile duct shown in Fig. 11 indicated that a comparatively large bile duct in the porta hepatis abruptly diminished in size and disappeared at several centimeters into the liver from the porta hepatis. In more peripheral portions of macroserial sections, small bile ducts were present. Severe cholangitis complicated following intestinal obstruction seemed to have destroyed and obliterated the intrahepatic bile ducts in this case.

Case 7. H.K., female. Her mother suffered from common cold at 3 months of pregnancy and took some drugs for this disease. The birth weight of the patient was $3,600 \mathrm{~g}$. The parents noticed persistent jaundice on the 24 th day of life. She was admitted to the Pediatrics, Tohoku University Hosptial on December 25, 1973 and referred to our clinic for operation. Total serum bilirubin was $12.8 \mathrm{mg} / 100 \mathrm{ml}$. The operation performed on the 87 th day of life revealed a total obliteration of extrahepatic bile ducts. The type of the biliary atresia was Kasai's III . Double-Y hepatic porto-jejunostomy with an external fistula was performed. The postoperative course was uneventful. Jaundice disappeared and total serum bilirubin was within a normal range 35 days after operation. However, she died of diffuse peritonitis due to perforation of strangulated intestine 4.5 months after the first operation. Autopsy revealed that the liver was comparatively soft and somewhat elastic. However, micro-abscesses were seen at portal tracts and there was no bile in the intestine. These findings suggested fresh complication of acute ascending cholangitis. Histological examination of the liver specimen disclosed moderate periportal fibrosis and ductular proliferation (Fig. 9). In this case, however, interlobular bile ducts were relatively large but showed inflammatory changes. The macroserial reconstruction of the liver showed the nearly normal structure of the biliary tree (Fig. 14). The bile ducts were well preserved throughout the liver in spite of evidence of severe inflammation.

Concerning the form of the extrahepatic bile duct in biliary atresia, several reports have been published (Kasai 1966; Gross 1953). Nowadays classification by these authors, especially Kasai's classification, has generally been accepted.

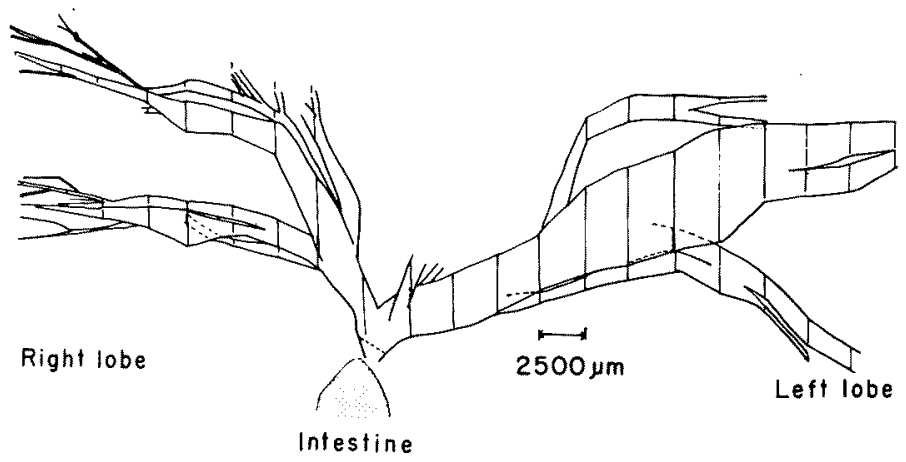

Fig. 14. A graphic reconstruction of the main bile ducts in Case 7 .

These findings are similar to those of the normal intrahepatic bile ducts. 
Furthermore, one of the authors (Kasai) described the histological findings of macroserial sections of extrahepatic bile ducts in his series of 68 cases and concluded that the obliterated portion of the bile duct showed similar histological features regardless of type and subtype of the lesion, or size of ducts (Kasai 1974). He also reported the histological findings of the bile duct in the region of porta hepatis and indicated a close correlation between size of the bile duct at the porta hepatis and postoperative excretion of bile. There have been several reports of microscopic study on surgical or autopsy specimens of the liver and a few reports on histological reconstruction of intrahepatic bile ducts (Oh-i et al. 1969; Landing et al. 1973). From reconstruction studies it has been shown that main interlobular bile ducts are usually open in the specimen taken from the peripheral portion of the liver. The study, however, has rarely been done on the patency of the intrahepatic biliary tree from the porta hepatis to the periphery of the liver of biliary atresia. The patency of bile ducts within the liver can be conjectured from clinical experiences in that active excretion of bile has been observed after bile duct-intestinal anastomosis in correctable type and after hepatic porto-enterostomy in non-correctable type of biliary atresia.

The result of the present study showed that the intrahepatic bile ducts were patent up to their peripheral portion in 6 of 7 cases of biliary atresia examined, regardless of the gross findings of extrahepatic bile ducts. Many anastomoses between main bile duct and proliferated ductules were confirmed by Oh-i et al, (1969) by means of histological reconstruction of interlobular bile ducts. It seems that bile draining ducts in the liver are patent at least in the early day of life. Some changes may occur, however, within the liver in the late stage which will result in obliteration of intrahepatic bile ducts. Bile plugs may be formed in intralobular bile ducts which obliterate bile flow in the tract. Progressive portal fibrosis may cause atrophy of interlobular bile ducts which may subsequently disappear. In our Case 5, it seems probable that severe ascending cholangitis complicated after successful operation destroyed the main bile ducts which might be finally obliterated by fibrous tissue.

Considering the results of the present study and reviewing the literature, it is concluded that operation should be done in the early days of life and that comparatively deeper dissection into the liver is preferable.

\section{References}

1) Gross, R.E. (1953) Obstructive jaundice in infancy. In: The Surgery of Infancy and Childhood, edited by R.E. Gross, Saunders Company, Philadelphia \& London, pp. 508-523.

2) Healey, J.E. (1953) Anatomy of the biliary ducts within the human liver. A.M.A. Arch. Surg., 66, 599-616.

3) Kasai, M. (1966) Surgery of liver and bile duct. In: Gendai Shonigaku Taikei Vol. 16 (Jap.), edited by Ts. Arakawa, T. Nagayama \& N. Yamada, Nakayama Co., Tokyo, pp. 104-139.

4) Kasai, M. (1974) Treatment of biliary atresia with special reference to hepatic portoenterostomy and its modifications. Prog. Pediat. Surg., 6, 5-52. 
5) Kasai, M., Asakura, Y., Suzuki, H. \& Ohashi, E. (1972) Modification of hepatic porto-enterostomy to prevent postoperative ascending cholangitis. In: Proceedings of the Fifth Annual Meeting of the Pacific Association of Pediatric Surgeons, edited by $\mathbf{K}$. Suruga PAPS Committee, Tokyo, p. 10.

6) Landing, B.H., Wells, T.R., Reed, G.B. \& Narayan, M.S. (1973) Disease of the bile ducts in children. In: The Liver, edited by B.H. Landing; The Williams \& Wilkins Co. Baltimore, pp. 480-509.

7) Oh-i, R., Kasai, M. \& Takahashi, T. (1969) Intrahepatic biliary obstruction in congenital bile duct atresia. Tohoku J. exp. Med., 99, 129-149.

8) Sawaguchi, S., Nakajo, T., Hori, T., Harada, Y. \& Ohbe, Y. (1968) Staged reconstruction of biliary tract for congenital bile duct atresia. Jap. J. Surg. (Jap.), 69, 13171318.

9) Suruga, K. (1970) Operations for biliary atresia. Shijutsu (Jap.), 24, 543-549. 